\title{
Memory Storage Difficulties from Partial Brain Damage
}

\section{Theofilidis Antonis*}

\author{
*Correspondence: Theofilidis Antonis
}

Address: Clinical Cognitive Neuropsychologist, MSc, Ph.D., 3rd University Psychiatric Clinic AUTh., Greece e-mail $\bowtie$ : antonis109@yahoo.gr

Received: 01 September 2020; Accepted: 10 September2020

Copyright: (C) 2020 Antonis T. This is an open-access article distributed under the terms of the Creative Commons Attribution License, which permits unrestricted use, distribution, and reproduction in any medium, provided that the original work is properly cited.

\section{ABSTRACT}

Introduction: Memory deficits, both short-term and long-term, as well as post-traumatic amnesia, are very common in patients who have undergone TBI, but rarely reflect a classic amnesia syndrome. Memory difficulties can be the result of many different factors, not the result of a single deficit. According to Brookshire (2007), reduced hippocampal volume and white matter are associated with memory deficits.

Aim: To investigate the neuropsychological profile and memory storage difficulties from partial brain damage in a case of post-traumatic epilepsy.

Material and Method: An international literature review was performed on Pub Med \& Cochrane online databases on epilepsy, and information material from the historical, psychiatric and neuropsychological evaluation of a female patient presenting with symptoms of mental disorder, history of epileptic seizures and history was used.

Results: The patient was shown to maintain a normal level of ability to perform daily activities as well as an assessment of reality control. Her performance across the range of cognitive abilities ranged below the normal limits for her age and level of education.

Conclusions: Development of psychosis following surgery to reduce seizures may be observed. Specific cognitive deficits and psychiatric symptoms are associated with impaired right temporal lobe function, and differentiate this particular patient profile from other syndromes associated with focal degeneration of the frontal and left temporal lobe.

Keywords: Memory Storage Difficulties, Partial Brain Damage, Epilepsy

\section{Introduction}

Psychiatric diseases are characterized as multifactorial and their cause is not fully understood as there are many underlying causes of the onset of psychopathology such as genetic background, life events, experiences, substance use, viral infections, etc. (Sidiropoulou, 2015). We know that epilepsy is caused by environmental or genetic factors. After a craniocerebral injury, for example, the brain in its attempt to correct the damage creates abnormal connections between cells that can cause epilepsy (Sidiropoulou, 2015). Epilepsy is considered a common disease and historically, it was associated with various prejudices and myths, as epilepsy was very easy, with sudden seizures, to be considered quite 
mysterious (Kay and Szaflarski, 2014). Hippocrates was one of the first doctors to emphasize that epilepsy can be treated, while its cause is attributed to disorders in the brain.

\section{Epileptic Seizures}

Seizures are one of the most common neurological symptoms occurring in human populations (Kay and Szaflarski, 2014). A seizure occurs due to abnormal, abnormal electrical activity in the brain. Seizures are defined as paroxysmal episodes caused by excessive, abnormal neuronal electrical discharges, located in specific areas of the brain or widely distributed (Kay and Szaflarski, 2014). Depending on the characteristics of the seizures and where they are located, they may or may not have observed clinical manifestations (Kay and Szaflarski, 2014). Abnormal stimulation and synchronization of neurons are basic conditions for the onset of seizures and are attributed to mechanisms that disrupt the depolarization and repolarization of membranes, as well as to a defective neural network. Seizures can disrupt many functions of the nervous system, causing abnormal motor, aesthetic, autonomic, behavioral and mental phenomena (Janz et al., 1985).

\section{The Temporal Lobes}

The temporal lobes are involved in the comprehension and memory of images as well as in the comprehension of language and emotions. They are responsible for hearing, perceiving complex images, understanding speech (in the left hemisphere) as well as behaviors that are responsible for motivation and emotion. Injuries to areas of the temporal lobe cause problems related to face recognition, word comprehension, selective attention, increased or decreased sexuality, as well as aggressive behavior, persistent speech (after damage to the right temporal lobe), olfactory and visual hallucinations, feelings of awe and panic. Accompanying motor phenomena include strange grimaces, chewing movements, etc. The most common cognitive deficits from trauma or seizures in the right temporal lobe involve working memory and facial cognition. Patients with lesions in this brain area experience behavioral problems such as social isolation, depressive symptoms and aggression.

Also in many cases they show hyper-religiosity, and strong visual illusions. The combination of clinical features associated with right temporal lobe dysfunction differs significantly from other syndromes associated with focal degeneration of the frontal and left temporal lobes and has thus been suggested to be considered a distinct variant of the frontal lobe.

\section{The Relationship Between Epilepsy and Psychosis}

Observations that seizures improved the psychotic symptoms of some patients led to the theory of functional dependence and biological competition for schizophrenic and epileptic symptoms. (Introduction of a method of creating iatrogenic seizures with drugs by von Meduna for the treatment of schizophrenia). Later Slater et al. By working with a small number of patients (69 people) they rejected 
the previous views and argued instead, the existence of a positive link between epilepsy and schizophrenia. The current position is that there is a relationship, but in the link that seems to have epilepsy and psychosis, there may be a rivalry of symptoms between seizures and psychotic symptoms (hallucinations and delusions).

Major Deficits as A Result of Traumatic Brain Injury (TBI)

All TBI cause serious effects, which are directly related to the rehabilitation, functionality and social reintegration of the individual. The person suffering from a head injury is expected to show various cognitive, psychosocial deficits, as well as difficulties in communication, speech and speech, which will appear in different forms and will depend on the percentage of damage, the point of damage, and by the individual himself. TBI, therefore, can significantly affect many sectors and deficits can range from very mild to very severe and improve or persist for many years or even remain throughout a person's life (Lezak et al., 2004). The main deficits that can be identified in a patient who has undergone TBI are analyzed below:

\section{Cognitive Deficits}

\section{Orientation}

Orientation is related to the awareness of the individual around four main axes: the individual, space, time and situations. People who have undergone TBI are often observed for the fact that they feel lost and disoriented in relation to time and space. Orientation presupposes the individual's ability to receive, store, and retrieve new information presented after injury. When the patient recovers with TBI, the recall of the conditions returns first and then the recall of space and time. Time is constantly changing, and therefore information should be constantly updated, presupposing an increased level of awareness (Brookshire, 2007).

\section{Attention Deficits}

Attention is a multifaceted cognitive function. This includes the individual's difficulties related to alertness, environmental awareness, selective attention, sustained and distracted attention. Vigilance and awareness refer to the state of consciousness in various sensory stimuli and the patient's perception in relation to the environment. Selective (or focused) attention generally refers to his ability to focus individual to one of many simultaneous stimuli, as well as the ability to resist distraction. Selective attention is thought to be controlled and directed by connections between the chamber and the prefrontal cortex. Sustained attention refers to a person's ability to maintain and focus on a stimulus for a period of time. Finally, distracted attention refers to the allocation of limited resources in multiple trials or procedures. (Brookshire, 2007). A slowing mental processing, in conclusion, is present in the attention deficits that occur in patients, including poor concentration, breakdown, and difficulty in 
accomplishing many different things at the same time (Sohlberg and Mateer, 2001). When attention problems are severe, the patient may complain of confusion, inability to think clearly, and disorientation.

Memory

Memory deficits, both short-term and long-term, as well as post-traumatic amnesia, are very common in patients who have undergone TBI, but rarely reflect a classic amnesia syndrome. Memory difficulties can be the result of many different factors, not the result of a single deficit. According to Brookshire (2007), reduced hippocampal volume and white matter are associated with memory deficits.

\section{Stimulus Response Time}

The slow way of reaction is a key characteristic of people who have undergone TBI. According to Brookshire (2007), these patients need more time to think before giving an answer. Especially in cases where the damage is diffuse, mental speed, memory and attention functions and the general cognitive performance of the patient are greatly degraded (Gronwall and Samson, 1994; Parker, 2001).

\section{Executive Functions}

Executive functions are a superior brain function, which contributes to the conquest and achievement of a goal. The wide range covered by these functions includes: inductive and productive thinking, flexibility, organizing and structuring actions, planning, problem-solving and strategyswitching, goal-setting, inhibiting and controlling emotional responses and behavior, as well as persistence in the test (Norman and Shallice, 1986; Brookshire, 2007). In cases of diffuse brain damage, patients tend to have poor performance on mental problems that need to be solved mentally (Grownwall \& Wrightson, 1981; Odgen, 1996). According to Marshall (1989), the effects of the cognitive and executive dysfunctions mentioned above can significantly impede communication.

\section{Immunoassay}

Patients who have undergone TBI are often unaware of their condition and are unaware of the deficits that exist, which is referred to as immunodiagnosis (Kay and Szaflarski, 2014). According to research, almost $40 \%$ of patients with TBI show immunodiagnosis, which is a significant obstacle to their development and recovery.

\section{Speech and Language Problems}

It is accepted that people who have suffered a head injury may present with the exception of cognitive and other deficits such as speech and language disorders. The most common deficits observed in speech are the following: 
- Disorganized and confused speech, both oral and written, which includes many inaccuracies, repetitions and revisions.

- Difficulty in finding the right words (lawlessness) and wrong naming.

- Hearing comprehension difficulties.

- $\quad$ Poor speech with short phrases and limited content.

- Semantic difficulties (vocabulary), reduced ability to use grammar and syntax correctly (syntax errors), at the level of oral and written speech.

- $\quad$ Aphasia Syndrome, if there are appropriate focal lesions and especially in cases of severe TBI (Sohlberg and Mateer, 1990).

\section{Communication}

Communication is defined as the exchange of information and messages between speakers (transmitter-receiver). Specifically, communication involves the transmission of a message from the transmitter's mind to the receiver's mind. In the literature, the "Message" is also referred to as communicative intention (Sabbagh, 1999). Communication can be either verbal or non-verbal (use of gestures and meanings). Patients with TBI may experience the following communication deficits:

- Difficulty in following a quick conversation.

- Difficulty watching a conversation in situations where the environment is not quiet and can distract the patient.

- $\quad$ Limited initiative to start and continue a discussion.

- $\quad$ Difficulty in understanding and interpreting a non-literal message (metaphor).

- Difficulty in understanding abstract concepts, sarcasm, oblique speech and indirect requests.

- $\quad$ Socially inappropriate use of language without inhibitions.

- Difficulty in identifying the appropriate communication environment and adapting the speech according to the communication context.

- Difficulty in drawing correct conclusions in all contexts. In conclusion, communication may be disrupted due to lack of logical content, lack of sensitivity to the needs and interests of the interlocutor, too much or too little information on the part of the patient and disturbed fact.

\section{Behavioral Changes}

Behavioral changes after a TBI are very common and have an impact on both the patient and his family members, friends and social environment. Behavioral problems range from irritability to psychotic behavior without inhibitions. The most common problems encountered in such patients are irritability, aggressive behavior, reduced anger control and management, impulsivity, lack of self- 
control, decreased social adaptability, disturbed social perception, emotional disturbances, egocentrism and depression. Depression is a consequence of persistent symptoms faced by the patient. Estimates of the prevalence of depression are around 35\% (Busch and Alpern, 1998). Fatigue, anxiety, change in the patient's pre-existing condition and irritability, tend to aggravate and intensify his depression, resulting in a vicious cycle that significantly delays the patient's recovery. In many cases, the patient with TBI, is possessed by a refusal to accept his problems and his condition and eventually leads to social withdrawal (Kay and Szaflarski, 2014). Behavioral and personality changes are much more often associated with long-term disability and tension in the patient's family and significant others than physical disability itself (Busch and Alpern, 1998). According to Matter (2001), these provocative and delinquent behaviors of a TBI patient may be associated with feelings of frustration and loss.

\section{Case Study}

The patient M.K. 49 years old, from Albania, she lives with her estranged husband. Reported onset of symptoms from the age of 31 (2001) when he had suffered a craniocerebral injury following a car accident (subdural hematoma of the temporal lobe, followed by craniotomy after four years at the age of 35). After the injury, "E" seizures, mixed anxiety and depression, outbursts of anger and impulsive behavior are reported. Worsening of symptoms has been reported for two years (she voluntarily discontinued her medication) with delusional ideas of persecution and counseling, irritability, aggression, auditory and visual hallucinations, lack of anesthesia and non-compliance with medication. In October 2018, she additionally presented behavioral disorganization (she set fire to the clothes of her husband). Its overall functionality after the accident is reported to be very low. No developmental disorders are reported from her personal memory. She has two adult children. No abuses are reported and she had two hospitalizations. The first was unintentional (March 2017) at the Psychiatric Hospital of Leros- Greece, lasting about two weeks due to behavioral disorganization and delusional ideas of persecution and counseling on the ground of post-traumatic epilepsy. The second hospitalization was voluntary at the University Psychiatric Clinic of AUTh, Greece, lasting one week in October 2018, with a diagnosis at the time of discharge: a) F06.2- organic (schizophrenic) delusional disorder, b) G98 - other disorders of the nervous system, not classified elsewhere. The medication he was receiving was as follows: 1) Zypadhera $405 \mathrm{mg}$ i.m. once a month, 2) tb Aloperidin $10 \mathrm{mg}$ 1/2-1 / 2-1, 3) tb Akineton $2 \mathrm{mg}$ 1-0-1,4) tb Stedon $10 \mathrm{mg}$ 1-1-1,5) tb Trileptal 300mg 1-1-1,6) tb Filicine 5mg 0-1-0. Her family psychiatric history as well as other medical history are freely mentioned.

\section{The Neuropsychological Assessment}

The evaluation of the cognitive function of the patient was carried out with the array of tests used in the neuropsychological laboratory of the C department University Psychiatric Clinic of AHEPA hospital, Thessaloniki-Greece. Her performance in each test was as follows: 


\section{Neuropsychological / Psychometric Tests}

1. MMSE: 24 (abnormal performance: <28)

2. MOCA: 16 (abnormal performance: <26)

3. Serial measurement: per3 up to $40 / 25^{\prime \prime} 4$ errors

4. Fast alphabet recitation: $61^{\prime \prime}$

5. Inverse numbering: 20-2: $12^{\prime \prime}$

6. Frontal Asseccment Battery (FAB): 08/30

7. Memory of episodes: $\mathrm{AD}=09 / 24 \mathrm{~s}, \mathrm{BD}=11 / 21 \mathrm{~s}$

8. Working memory: forward $=$ recall 4 numbers $/$ backwards $=$ recall 3 numbers

9. Verbal flow test: 06 Animals, 09 Fruits, 03 Words from X / 60"

10. Trail Making Test: $A^{\prime}=135^{\prime \prime}$ and 5 errors, $B^{\prime}=$ NO

11. Coding: 33 / 120"

12. Optical-spatial ability test: out of normal range

13. Clock test: Marginal normal limits

14. Naming test: $78 / 100$

15. REY FIGURE: $26 / 32$ copy

16. STROOP: reading: 13 errors $/ 84^{\prime \prime}$, naming: 7 errors $/ 51^{\prime \prime}$, interpolation: NO

17. Eupraxia: (hammer, comb, scissors) $3 / 3$

18. Speech flow: Normal.

19. Paraphrases of words: No.

20. Phonological paraphrases: Some

21. Perception of oral speech: Good

22. Repetition of oral speech: Good

23. Perception of written language: Good

24. Reading aloud: Normal with errors

25. Scripture: Normal

\section{Evaluation}

According to the results of the tests at the time, the patient showed a characteristic cognitive dysfunction with deficits in the ability of intermittent attention, coding and information processing speed, learning, functional (working) memory and executive function. In particular, memory deficits occurred in tests requiring free recall, interpolation, and working memory, while reduced attention deficit was found to be directly related to difficulty concentrating on a task. Delusional persecution ideas as well as depressive symptoms were reported by the patient. The overall deficit score obtained from 
the examination is related to her medical history according to which at the age of 31 she suffered TBI in a car accident and at the age of 35 she underwent a craniotomy to remove subdural hematoma in the right temporal lobe associated with seizures (post-traumatic epilepsy).

\section{Neuropsychological Assessment}

The main goal of the neuropsychological assessment of the patient M.K. was the investigation mainly of her cognitive deficits with the basic set of tests of the neuropsychological laboratory of the $\mathrm{C}$ department University Psychiatric Clinic of AUTh university Greece, which examines the major points of cognitive behavior and performance. Specifically assessed: levels of attention and concentration, visual perception, learning ability, parameters of memory (verbal, visual, working, long-term), verbal functions and academic skills, visual-spatial and visual-constructive ability, abstract thinking, information processing speed, ability to form concepts, and executive functions. From the patient's history did not arise in the first year the need to go beyond the basic array of assessment of cognitive abilities and add specialized tests. The neuropsychological examination included tests to assess memory function (Story memory, reverse number retrieval, verbal flow test), attention (stroop, direct number recall, Trail Maiking Test A), and executive functions (Trail Maiking Test B, Cube test). Her score on both immediate and long-term memory tests (story memory, reverse number retrieval, verbal flow test) appeared deficient for her age and cognitive level. Regarding the long-term retention at the specific time of the evaluation, difficulties were observed in the tests that allow the conceptual organization of the data to be memorized (story memory). The maintenance of mnemonic traces concerning general knowledge (declarative memory) fluctuated at normal levels. The patient's attention span fluctuated at very low levels as indicated by the slow execution of the A part of the Trail Maiking Test but also by its performance in the Stroop interpolation condition. In general, the patient's performance in information processing speed, learning, memory and executive function ranged below the normal limits for her age and level of education at that time. In particular, the patient showed reduced accuracy and speed of rotation of visual attention to a different type of reaction, difficulty in abstract thinking and parallel processing of information according to its performance in the individual tests of the neuropsychological array. The patient had obvious difficulty in the Speech Flow Test (Speech Flow Index) (6 reports / 60" in the "ANIMALS" category and 9 reports / 60" in the "FRUIT" category).

In particular, particular deficiencies were found in vigilance (ability to respond), as well as in vigilance (general receptivity to stimuli and readiness for response). Deficits in the dimensions of both immediate and long-term memory were characteristic. The patient showed that she maintains at normal levels the ability to perform daily activities as well as to assess / control reality. 


\section{Conclusions}

The appearance of cognitive deficits associated with post-traumatic epilepsy of the right temporal lobe, verifies the relevant neurocognitive profile in the case of the patient MK. The patient had memory problems and could not retrieve information when she needed it. Also her attention was affected with the immediate result of slowing down the speed of information processing. We know that memory depends to a large extent on attention. The deficient attention of the patient MK seemed to have consequences in her memory as well. People with epilepsy, even if they can encode the information, may not remember it due to storage-dependent difficulties in brain systems that may have suffered from partial damage. The patient also showed problems in solving problems related to the reproduction of contents. In particular, it showed that it could not combine different contents of information. The specific patient with temporal lobe epilepsy presented special and characteristic deficits in the recall of episodic memory due to problems in attention, organization and delimitation of information, reduced cognitive flexibility, behavioral disorganization, removal of inhibitions, delusions and delusions. compliance with the medication and the instructions of her treating physician. This patient case supports the view that some patients develop psychosis after epilepsy surgery and that the combination of clinical features associated with right temporal lobe dysfunction differs significantly from other syndromes associated with focal frontal degeneration and of the left temporal lobe and can be considered as a distinct syndrome with highly cognitive and behavioral deficits.

\section{References}

Acharya MM, Hattiangady B, Shetty AK. Progress in neuroprotective strategies for preventing epilepsy. Prog Neurobiol 2008; 84: 363-404.

Brookshire RH. Introduction to Neurogenic Communication Disorders, 7th edition. St Louis: Mosby Elsevier; 2007.

Busch CR and Alpern HP. Basal salivary cortisol levels in patients suffering from Postconcussion Syndrome. Unpublished data; 1998.

Gronwall D and Wrightson P. Memory and information processing capacity after closed head injury. J Neurol Neurosurg Psychiatry 1981; 44: 889-895.

Janz NK, Becker MH, Hartman PE. Contingency contracting to enhance patient compliance: a review. Patient Educ Couns 1984; 5: 165-178.

Kay B and Szaflarski JP. EEG/fMRI contributions to our understanding of genetic generalized epilepsies. Epilepsy Behav 2014; 34: 129-135.

LaPointe LL. Aphasia and related neurogenic language disorders (4th ed.) New York 2011. NY: http:books.google.com/books

Lezak MD, Howieson DB, Loring DW. Neuropsychological assessment (4th ed.) New York.: Oxford University Press; 2004.

Norman DA and Shallice T. Attention to Action: Willed and Automatic Control of Behaviour. In: Davidson RJ, Schwartz GE, \& Shapiro DE. (Eds.), Consciousness and Self-Regulation. New York: Plenum Press; 1986: 1-14.

Ogden JA. Fractured minds: A case-study approach to clinical neuropsychology. New York, NY, US: Oxford University Press; 1996.

Parker IM. Invasion dynamics of Cytisus scoparius: a matrix model approach. Ecol Appl 2001; 10: 726-743. 
Sabbagh MA. Communicative Intentions and Language: Evidence from right hemisphere damage and autism. Brain Lang 1999; 70: 29-69.

Slater A, von der Schulenburg C, Brown E, Badenoch M, Butterworth G, Parsons S, Samuels C. New- born infants prefer attractive faces. Infant Behav Dev 1998; 21: 345-354.

Sohlberg MM and Mateer CA. Cognitive Rehabilitation: An integrative neuropsychological approach. New York, NY: Guilford; 2001. 